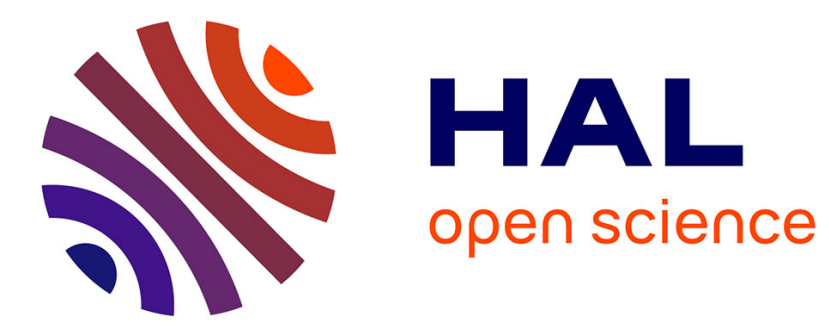

\title{
Monolithic Fluid Structure Interaction Model, Application to Water Entry Problem
}

\author{
Frederic Golay
}

\section{To cite this version:}

Frederic Golay. Monolithic Fluid Structure Interaction Model, Application to Water Entry Problem. Topical Problems of Fluid Mechanics 2018, Feb 2018, Prague, Czech Republic. 10.14311/TPFM.2018.016 . hal-01805263

\section{HAL Id: hal-01805263 \\ https://hal.science/hal-01805263}

Submitted on 10 Dec 2021

HAL is a multi-disciplinary open access archive for the deposit and dissemination of scientific research documents, whether they are published or not. The documents may come from teaching and research institutions in France or abroad, or from public or private research centers.
L'archive ouverte pluridisciplinaire HAL, est destinée au dépôt et à la diffusion de documents scientifiques de niveau recherche, publiés ou non, émanant des établissements d'enseignement et de recherche français ou étrangers, des laboratoires publics ou privés. 


\title{
MONOLITHIC FLUID STRUCTURE INTERACTION MODEL, APPLICATION TO WATER ENTRY PROBLEM
}

\author{
F. Golay ${ }^{1}$ \\ ${ }^{1}$ Université de Toulon, IMATH, CS 60584, 83041 Toulon Cedex 9, France
}

\begin{abstract}
We consider the interaction between an air-water flow and a rigid structure using a fast accurate numerical model. Following the 3D low Mach compressible Euler model previously developed and validated, a compressible fluid-structure interaction model is proposed. In the context of a fictitious domain, a volumic penalization is applied inside the body to ensure a rigidity constraint through a penalized velocity in order to get the correct motion of the rigid body. The accuracy of this simple model is improved using block-based adaptive mesh refinement technique. The improvement and the validity of our fluid-structure interaction procedure is investigated through the numerical simulation of the water entry of a cylinder freely falling and impacting the water. This test case is compared to experimental data and other numerical simulations.
\end{abstract}

Keywords: Euler equations, low Mach, compressible, bi-fluid, mesh refinement, fluid-structure interaction, fictitious domain, penalization, projection method, interface-sharpening.

\section{Introduction}

We consider fluid-structure interaction (FSI) in the context of coastal engineering. From previous work, we have proposed a powerful model to simulate air-water flow [11]. This model is based on a low Mach compressible approach which solves Euler equations in two phases (air/water), where the viscous term in the Navier-Stokes equation is neglected. The model is referred as an artificial compressibility model since it uses an non-physical speed of sound using an isothermal equation of state. This allows a less restrictive time-step for faster simulations. In order to get both fast and accurate results, an adaptive mesh refinement has been designed to refine area of interest, especially around a structure embedded in the flow and everywhere it is necessary. The numerical production of entropy is used as mesh refinement criterion. This approach has been validated and confronted to many hydrodynamic problems [19, 9, 10, 2].

FSI problems are encountered in a wide range of problems and various methods are available to take into account the presence of an obstacle in a flow. Mittal and Iaccarino [16] give a relevant detailed review of such methods. For sake of simplicity, we have chosen to implement a penalization method. This method has been presented by Coquerelle and al. [6] in the case of incompressible flows. Following [5], we propose the formulation in the case of compressible flows.

In order to validated this formulation, we present the numerical simulation of water entry problem. Indeed, the interaction between free surface and rigid body is of main interest in ocean engineering: floating structure, sloshing, green water loading on deck, objects dropping or rising onto water surface. This leads to many experiments in the case of wedge or cylinder falling into water $[12,13,7]$ and numerical simulations $[24,15,22,23,20,14]$. Here we propose to investigate the water entry of a cylinder freely falling and impacting the water as described experimentally by Greenhow and Lin [12] and we compare our numerical simulations with [22, 23]. The first part of the paper recalls the air-water model and its numerical formulation using finite volume in adaptive mesh refinement framework. Then, the fluid-structure interaction model is presented. The last part of the paper is devoted to the validation of the FSI model, through the numerical simulation of the water entry problem of a circular cylinder. 


\section{Two-fluid low Mach compressible Euler model}

\subsection{Governing equations}

We consider an inviscid isothermal compressible flow ruled by Euler equations with a mixture of air and water [11]. The mass and momentum conservation equations give :

$$
\frac{\partial \rho}{\partial t}+\nabla \cdot(\rho \mathbf{u})=0
$$

and

$$
\frac{\partial(\rho \mathbf{u})}{\partial t}+\nabla \cdot(\rho \mathbf{u} \otimes \mathbf{u}+p \overline{\overline{\boldsymbol{I}}})=\rho \mathbf{g},
$$

where $\rho$ denotes the density, $p$ the pressure and $\mathbf{u}$ the velocity. The volume fraction $\phi$ follows an advection equation written in non-conservative form in order to avoid spurious oscillations at the interface air-water [11] :

$$
\frac{\partial \phi}{\partial t}+\mathbf{u} \cdot \nabla \phi=0
$$

Equations (1), (2) and (3) form a hyperbolic system which is closed with an artificial pressure law,

$$
p=p_{0}+c_{0}^{2}\left(\rho-\left(\phi \rho_{A}+(1-\phi) \rho_{W}\right),\right.
$$

where $p_{0}$ is the reference pressure, $c_{0}$ speed of sound in the mixture and $\rho_{A}$ and $\rho_{W}$ the density of air and water respectively. The artificial speed of sound of the mixture is taken much below the physical expected value in order to avoid a too restrictive time step due to the explicit scheme in time and to get a Mach number about 0.1 compatible with almost incompressible flow.

\subsection{Numerical implementation}

The numerical resolution of the hyperbolic system is based on a Godunov-type finite volume scheme. We use an explicit second order Runge-Kutta time integration's scheme. The spatial discretization is improved using the second order Monotonic Upwind Scheme for Conservation Laws reconstruction scheme (MUSCL) with Barth-Jespersen limiter. The equation (3) is known to be numerically very diffusive. Following [21], a mass-conserving interface-sharpening procedure has been applied to counteract the effect of the numerical diffusion, which lead us to solve a corrective system of equations by means of a splitting procedure [2]:

$$
\begin{aligned}
& \frac{\partial \rho}{\partial \tau}=\phi^{2}(1-\phi)^{2}(\phi-c)\left(\rho_{A}-\rho_{W}\right) \\
& \frac{\partial \rho \mathbf{u}}{\partial \tau}=\phi^{2}(1-\phi)^{2}(\phi-c) \mathbf{u}\left(\rho_{A}-\rho_{W}\right), \\
& \frac{\partial \phi}{\partial \tau}=\phi^{2}(1-\phi)^{2}(\phi-c),
\end{aligned}
$$

where $c$ is computed numerically to insure mass conservation, i.e.:

$$
c=\frac{\int_{\Omega} \phi^{3}(1-\phi)^{2} \mathrm{~d} \Omega}{\int_{\Omega} \phi^{2}(1-\phi)^{2} \mathrm{~d} \Omega} .
$$

\subsection{Mesh refinement strategy}

A dynamic block based adaptive mesh refinement method (BB-AMR) with a local multi-time step algorithm has been developed to balance fast computations and accurate results. The computational domain is divided in multiple blocks and each of them can be meshed according to its refinement. In general, considering a hyperbolic system of conservation law of the form ,

$$
\frac{\partial(\mathbf{w})}{\partial t}+\nabla \cdot \mathbf{f}(\mathbf{w})=0
$$


where $\mathbf{w}$ denotes the conservative variables and $\mathbf{f}$ the conservative flux, as for the one we use here, a mathematical entropy can be derived, veryfing the Lax's entropy condition :

$$
\frac{\partial s(\mathbf{w})}{\partial t}+\nabla \cdot \psi(\mathbf{w}) \leq 0
$$

where the entropy flux $\psi(\mathbf{w})$ satisfy $\nabla_{\mathbf{w}}^{T} \psi=\nabla_{\mathbf{w}}^{T} s(\mathbf{w}) \mathbf{f}^{\prime}(\mathbf{w})$. In the present case, for the two-fluid model, the expression of entropy and entropy flux can be :

$$
\begin{gathered}
s=\frac{1}{2} \mathbf{u}^{2}+c_{0}^{2} \rho \ln \rho-c_{0}^{2}\left(\rho_{W}-\rho_{A}\right) \phi, \\
\psi=\left(\frac{1}{2} \rho \mathbf{u}^{2}+c_{0}^{2} \rho(\ln \rho+1)\right) \mathbf{u} .
\end{gathered}
$$

The numerical entropy production is used as mesh refinement criterion for each block dividing the computational domain. Locally, the block is coarser or finer according to the value of its numerical production of entropy relative to the numerical production of entropy of the whole domain. More details can be found in $[9,10,2]$. The interest of such a criterion is that the entropy acts both as an indicator error as well as a indicator of locations where shocks occur (ie. areas that need to be refined) as mentionned in [17] and [8]. Contrarily to previous domain decomposition method based on Cuthill-McKee scheme [2], which is time consuming, now Morton's code is applied to the block numbering. Indeed, using space filling curves in order to renumber the blocks allows to browse all blocks preserving their locality (see e.g. [18, 1]). Following the space filling curve, each block is affected to a domain (or MPI process) according to a predefined number of cells. It insures well balanced process and, most of the time, lower domain interfaces (lower process communications). The Z-order numbering is very easy to implement and is computed just once. The spatial coordinates of the block's center are transformed into integer and translated in binary. Then, the Morton code consists on interleaving the binary identification in order to form a single binary number. For example, let us consider the triplet $(2,5,0)_{10}$ translated in binary $(101,101,000)_{2}$, then interleaved $(010100010)_{2}$ and finally it corresponds to the number 162 . An example of Z-order curve and domain decomposition is presented Fig. 1.
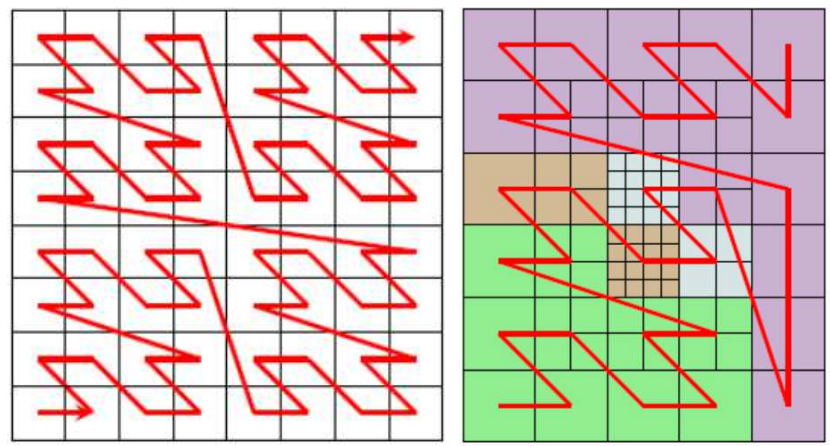

Figure 1: Examples of Morton numbering: Four iteration of the Zorder curve (left) and application to AMR mesh decomposed in 4 domains (right).

\section{Fluid-structure interaction}

Among all methods available to take into account an obstacle in a flow, we seek the one which allows us both an easy and time-saving procedure. Following [6], we choose a monolithic approach and consider the fluid-solid system as unified. The penalization method consist in considering the rigid body as a particular "rigid-fluid". The idea is to add a source term in the continuity and momentum equation in order to impose the density of the solid $\rho_{s}$ and a velocity field compatible with the motion of a rigid solid $\overline{\mathbf{u}}$. Introducing the Heaviside function $\chi$ ( $\chi$ equals 1 inside the 
solid and 0 otherwise) and a penalization parameter $\lambda$, the formulation of the penalized problem can be formulated as:

$$
\begin{gathered}
\frac{\partial \rho}{\partial t}+\nabla \cdot(\rho \mathbf{u})=\chi \lambda\left(\rho-\rho_{s}\right) \\
\frac{\partial(\rho \mathbf{u})}{\partial t}+\nabla \cdot(\rho \mathbf{u} \otimes \mathbf{u}+p \overline{\overline{\boldsymbol{I}}})=\rho \mathbf{g}+\chi \lambda \rho_{s}(\mathbf{u}-\overline{\mathbf{u}}) .
\end{gathered}
$$

Numerically, the Heaviside function is determined using a ray-casting algorithm [3]. In fluid, the last term of equation (8) vanishes and one recovers Euler equations. Inside the solid, the last term of the equation (8) exists and its contribution is weighted by $\lambda . \quad \lambda$ is chosen very large so that $\lambda \rho_{s}(\mathbf{u}-\overline{\mathbf{u}})$ becomes dominant and it remains $\mathbf{u}=\overline{\mathbf{u}}$, where $\overline{\mathbf{u}}$ is the velocity we want to apply in the solid and will be defined below. Numerically, the implementation of the penalization seems simple as only an additional source term has to be added. One needs to be careful about the value of the penalization parameter since it must be chosen relative to the time step in order to keep stability because of the explicit time scheme. However, as pointed out in [6], the fluid structure problem can be solved by solving successively the Euler equations and a "correction" equation through time-splitting. This correction corresponds to the projection of the velocity field onto a "rigid" velocity field. More details can be found in [4].

To find the penalization velocity relative to the velocity of the fluid, the latter is projected onto a "rigid" velocity field, that is a velocity that verifies :

$$
\overline{\mathbf{u}}=\mathbf{V}_{\mathbf{G}}+\boldsymbol{\omega} \times \mathbf{G M}
$$

where $\times$ denotes the cross product, $\mathbf{V}_{\mathbf{G}}$ is the translation velocity, $\boldsymbol{\omega}$ the angular velocity and $\mathbf{G M}$ the vector between $\mathrm{G}$ the center of gravity of the solid and an arbitrary point in the solid.

The projection can be obtained by minimizing the following functional over the solid domain $\Omega$ :

$$
\min J=\int_{\Omega}\left(\rho \mathbf{u}-\rho_{s} \overline{\mathbf{u}}\right)^{2} \mathrm{~d} \Omega .
$$

In the case where $\rho_{s}$ is non uniform, the translation velocity and angular velocity are determined solving:

$$
\begin{gathered}
\left(\int_{\Omega_{s}} \rho_{s}^{2} \mathrm{~d} \Omega\right) \mathbf{V}_{\mathbf{G}}+\boldsymbol{\omega} \times\left(\int_{\Omega_{s}} \rho_{s}^{2} \mathbf{G} \mathbf{M} \mathrm{d} \Omega\right)=\int_{\Omega_{s}} \rho \rho_{s} \mathbf{u} \mathrm{d} \Omega \\
\left(\int_{\Omega_{s}} \rho_{s}^{2} \mathbf{G M} \mathrm{d} \Omega\right) \times \mathbf{V}_{\mathbf{G}}+\left(\int_{\Omega_{s}} \rho_{s}^{2}\left(\mathbf{G M}^{2} \mathbf{I}_{\mathbf{d}}-\mathbf{G M} \otimes \mathbf{G M}\right) \mathrm{d} \Omega\right) \cdot \boldsymbol{\omega}=\int_{\Omega_{s}} \rho \rho_{s} \mathbf{G M} \times \mathbf{u} \mathrm{d} \Omega
\end{gathered}
$$

where $\mathbf{I}_{\mathbf{d}}$ denotes the identity matrix and $\otimes$ the tensorial product. In the case where $\rho_{s}$ is uniform, the translation velocity and angular velocity are simply determined by:

$$
\mathbf{V}_{\mathbf{G}}=\frac{1}{|M|} \int_{\Omega} \rho \mathbf{u} \mathrm{d} \Omega,
$$

and

$$
\boldsymbol{\omega}=\overline{\overline{\mathbf{J}}}^{-1} \int_{\Omega} \mathbf{G M} \times \rho \mathbf{u} \mathrm{d} \Omega,
$$

where $\overline{\overline{\mathbf{J}}}^{-1}$ is the inverse of the inertia matrix of the solid and $M$ its mass.

\section{Water entry of a circular cylinder}

Greenhow and Lin did a drop experiment of horizontal cylinder in calm water [12]. They considered a cylinder of diameter $0.11 \mathrm{~m}$, density $500 \mathrm{~kg} / \mathrm{m}^{3}$ (half buoyant cylinder) and another one, with the same diameter, but a density of $1000 \mathrm{~kg} / \mathrm{m}^{3}$ (neutrally buoyant cylinder). This experiment was many times used to validate numerical simulations. As in [22], we consider a water height of $0.65 \mathrm{~m}$, and we chose a air height of $1,35 \mathrm{~m}$ over a width of $2 \mathrm{~m}$, in order to reduce the influence of 
the mirror boundary conditions imposed everywhere. The cylinder, with zero velocity, is dropped from a height of $0.5 \mathrm{~m}$, which is measured from the centre of the cylinder at rest to the calm water surface. The computational domain is divided in $32 \times 32$ blocks composed of one cell. The maximum level of mesh refinement is 5. It means that the maximum number of cells per block is $\left(2^{5}, 2^{5}, 1\right)$, which corresponds to a mesh size of $1.9 \mathrm{~mm}$. Initially, the mesh is refined at the maximum level around the cylinder and at the air-water interface. During the computation, the mesh is adapted according to the numerical production of entropy. Let $\bar{P}_{\omega}$ (resp. $P_{b}$ ) denotes the average numerical production of entropy over all blocks (resp. one block), then if $P_{b}<\alpha_{\min } \bar{P}_{\Omega}$ the block is coarsen and if $P_{b}>\alpha_{\min } \bar{P}_{\Omega}$ the mesh is refined. With $\alpha_{\min }=20 \%$ and $\alpha_{\max }=40 \%$ the initial mesh is composed of more than 200000 cells and then around 80000 cells as presented Fig. 2.

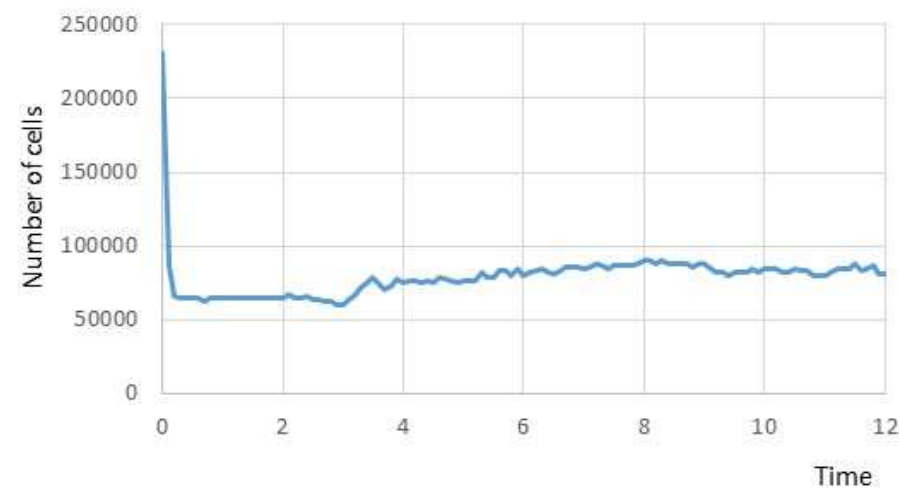

Figure 2: Number of cells during the adaptive mesh refinement procedure.

The computation, over 8 domains (or Mpi processes) run for 2 hours on one single IntelCoreI7 processor for a real time simulation of 1.2 seconds. The boundary of the cylinder, which circumference is discretized in 64 parts, is meshed with triangle faces in order to define the heaviside function $\chi$. On Fig. 3, the position of the centre of the cylinder is plotted during time. The cylinder impact the water at $t=0.3 \mathrm{~s}$. As the cylinder drop in the air, the variation of density is not important and the curves are superposed. After, the half buoyant cylinder naturally rise the surface. The calculated results for the motion of the cylinder into the water are compared with the experimental data by Greenhow and Lin [12], and reasonable agreement is shown.

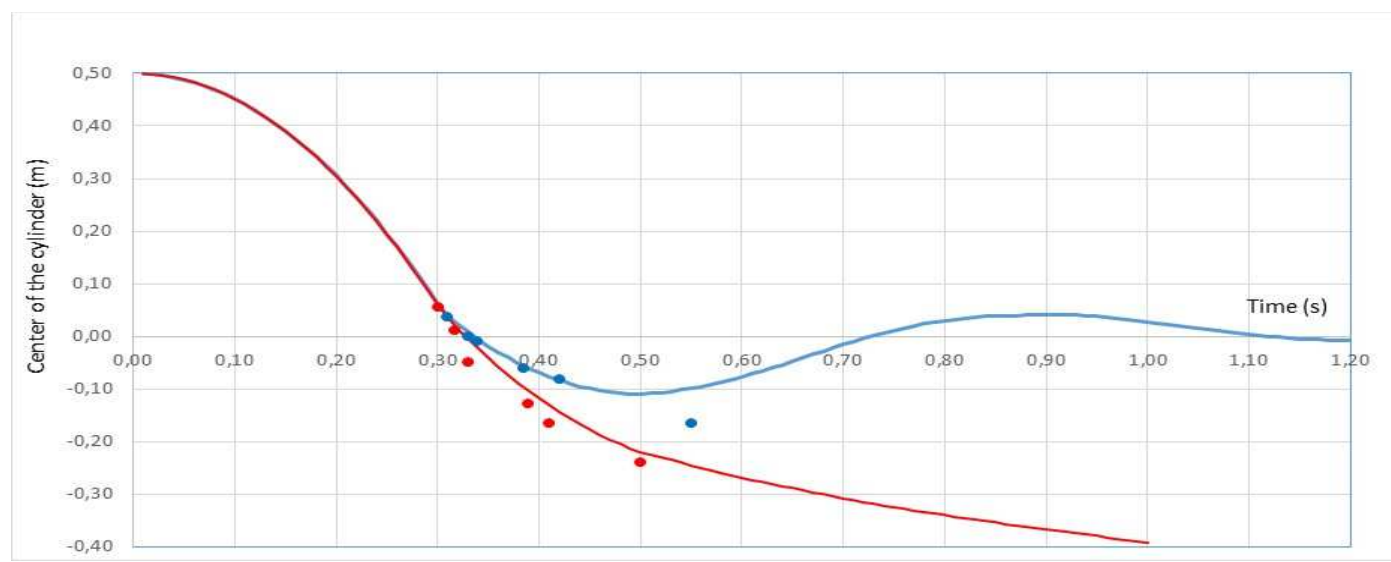

Figure 3: Position of the center of the cylinder during time. Red neutral buoyant cylinder, blue half buoyant cylinder. Solid line numerical simulation, point experiment.

The free surface profiles at $t=0.385 \mathrm{~s}$ for the half buoyant cylinder is shown Fig. 4. Our 
simulation (figure at left and red solid line at right) is compared to the experimental result of Greenhow and Lin [12] and to the result of Sun and Faltinsen [22] (green solid line) using boundary element method (BEM) . We found a good agrement. One can notice that, thanks to the interface sharpening procedure (5), the thickness of air-water interface is lower than 3 cells.
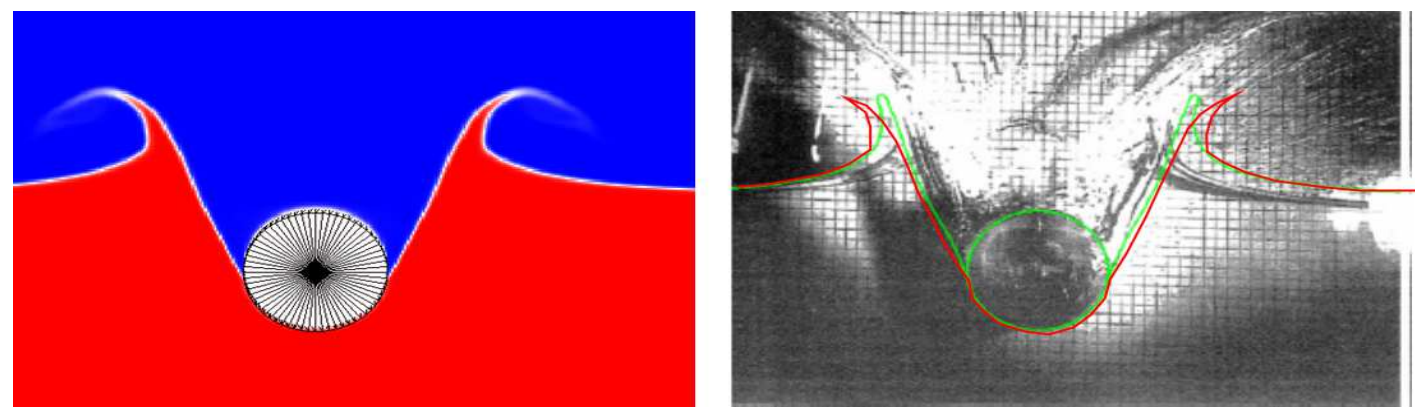

Figure 4: Penetration of the cylinder at $t=0.385 \mathrm{~s}$. Left: present numerical simulation. Right: Experiment [12], free surface profile of present numerical simulation with red solid line, free surface profile of BEM results by [22] with green solid line.

Fig. 5, water entry of half buoyant cylinder at $t=0.37 \mathrm{~s}, t=0.63 \mathrm{~s}, t=0.77 \mathrm{~s}$ are presented. On the left, according to the block based adaptive mesh refinement procedure the mesh and the numerical production of entropy are presented. In the middle the density is plotted and describe the free surface profiles. This results are compared to the results of Sun and al. [23] using weakly compressible Smoothed particle hydrodynamics (SPH) method. We found also a good agreement.
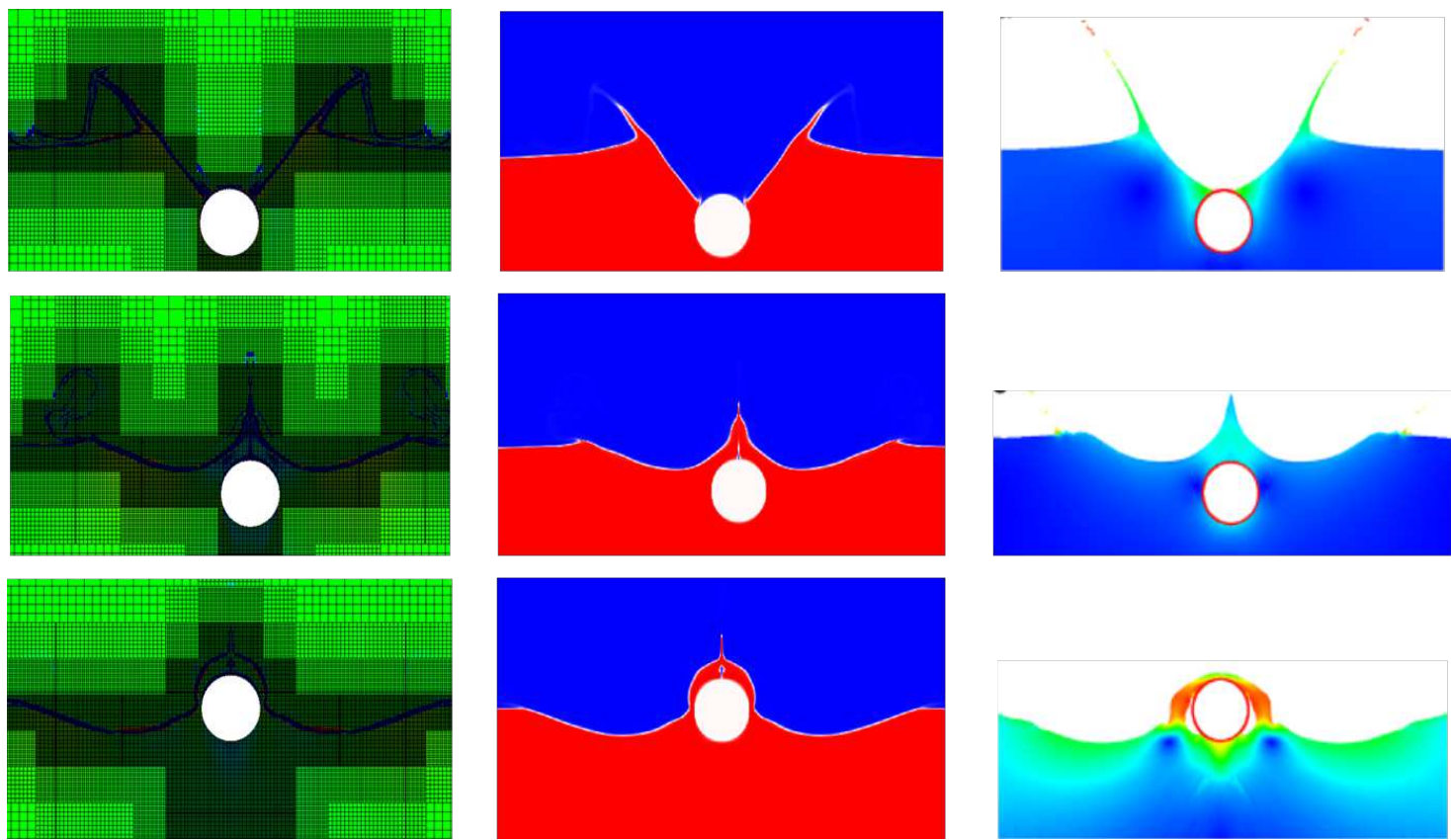

Figure 5: Water entry of half buoyant cylinder at $t=0.37 s, t=0.63 s, t=0.77 s$. Left: mesh and numerical production of entropy (green: zero, blue: negative value). Middle: density (red:water, blue:air). Right: results of Sun and al. [23] using SPH method. 


\section{Conclusion}

The aim of this study was to propose a fast and reliable procedure to treat fluid-structure interaction from an existing weakly compressible air-water model. By considering a fictitious domain approach, we have developed a volumic penalization based on a prediction-correction method. The body's motion is imposed by a penalization velocity which is computed according to the flow's dynamic. This approach was confronted to the water entry problem of a cylinder freely dropping into calm water. The results were successfully compared to experimental and numerical results. The fluid-structure solver has greatly benefited from Block Based Adaptive Mesh Refinement procedure and can be considered as a good compromise between accuracy and time computing.

\section{Acknowledgments}

This work has been supported by the Region Provence Alpes Côte d'Azur (PACA).

\section{References}

[1] F. Alauzet and A. Loseille, On the Use of Space Filling Curves for Parallel Anisotropic Mesh Adaptation, Proc. the 18th Int. Meshing Roundtable, (2009), pp. 1-18.

[2] T. Altazin, M. Ersoy, F. Golay, D. Sous and L. Yushchenko, Numerical investigation of BB-AMR scheme using entropy production as refinement criterion, Int. J. Comp. Fluid Dyn., 30-3 (2016), pp. 256-271.

[3] T. Altazin, F. Golay and P. Fraunie, A 3D unified model to Fluid-Structure Interaction with Block Based Adaptive Mesh Refinement, Proc. Topical Problems of Fluid Mechanics, (2016), pp. 1-8.

[4] T. Altazin, Un modèle d'interaction fluide-structure en régime compressible faible Mach, Phd university of Toulon (in french), (2017).

[5] T. Altazin, F. Golay And P. Fraunie, A 3D unified model to Fluid-Structure Interaction with Block Based Adaptive Mesh Refinement, Proc. 26st International Offshore and Polar Engineering (ISOPE), Rhodes, Greece, (2016), pp. 1-8.

[6] M. Coquerelle And G.-H. Cottet, A vortex level set method for the two-way coupling of an incompressible fluid with colliding rigid bodies, J. Comp. Phys., 227 (2008), pp. 9121-9137.

[7] G. Colicchio, M. Greco, M. Miozzi, And C. Lugni, Numerical investigation of the waterentry and water-exit of a circular cylinder, In 24th Intl. Workshop on Water Waves and Floating Bodies, Zelenogorsk, Russia, April 1922, (2009).

[8] J.-P. Croisille, Contribution à l'étude théorique et à l'approximation par éléments finis du système hyperbolique de la dynamique des gaz multidimensionnelle et multiespèces, $\mathrm{PhD}$ thesis, Paris 6, 1990.

[9] M. Ersoy, F. Golay, And L. Yushchenko, Adaptive multiscale scheme based on numerical density of entropy production for conservation laws, Open Mathematics, 11 (2013), pp. 13921415.

[10] F. Golay, M. Ersoy, L. Yushchenko, and D. Sous, Block-based adaptive mesh refinement scheme using numerical density of entropy production for three-dimensional two-fluid flows, Int. J. Comput. Fluid Dyn., 29 (2015), pp. 67-81.

[11] F. Golay And P. Helluy, Numerical schemes for low Mach wave breaking, International Journal of Computational Fluid Dynamics, 21 (2007), pp. 69-86.

[12] M. Greenhow And W.-M. Lin, Nonlinear free surface effects: experiments and theory, MIT Internal Report, Tech. Rep. 83-19, (1983), pp. 1-95. 
[13] M. Greenhow, Wedge entry into initially calm water, App. Ocean Res., 9-4, (1987), pp. 214223.

[14] A. Kiara, R. Paredes And D.K.P.. Yue, Numerical investigation of the water entry of cylinders without and with spin, J. Fluid. Mech., 814 (2017), pp. 131-164.

[15] K.M. T. Kleefsman, G. Fekken, A. E.P. Veldman and B. Iwanoski, An improved volume-of-fluid method for wave impact problems, Proc. International Offshore and Polar Engineering (ISOPE), Toulon, France, (2004), pp. 334-341.

[16] R. Mittal and G. Iaccarino, Immersed boundary methods, Annu. Rev. Fluid Mech., 37 (2005), pp. 239-261.

[17] G. Puppo, Numerical entropy production on shocks and smooth transitions, J. sci. comp., 17 (2002), pp. 263-271.

[18] S.M.. Ruffin And J. LeE, Implementation of a 3D Hilbert SFC into a Parallel CartesianGrid Flow Solver ,Int. J. Math. Models Meth. App. Sci., 7-6 (2012), pp. 803-810.

[19] A. N. Sambe, D. Sous, F. Golay, P. Fraunié, and R. Marcer, Numerical wave breaking with macro-roughness, Eur. J. Mech.-B/Fluids, 30 (2011), pp. 577-588.

[20] M. Sasson, S. Chai, G. Beck, Y. Jin And J. Rafieshahraki, A comparison between Smoothed-Particle Hydrodynamics and RANS Volume of Fluid method in modelling slamming, J. Ocean Eng. Sci., 1, (2016), pp. 119-128.

[21] K.-M. SHyUE, An Eulerian interface-sharpening algorithm for compressible gas dynamics, in Modeling, Simulation and Optimization of Complex Processes-HPSC 2012, Springer, 2014, pp. $221-231$.

[22] H. Sun And O.M. Faltinsen, Water impact of horizontal circular cylinders and cylindrical shells, App. Ocean Res., 28, (2006), pp. 299-311.

[23] P. Sun F. Ming And A. Zhang, Numerical simulation of interactions between free surface and rigid body using a robust SPH method, App. Ocean Res., 98, (2015), pp. 32-49.

[24] R. Zhao and O. Faltinsen, Water entry of two-dimensional bodies, J. Fluid. Mech., 246 (1993), pp. 593-612. 